\title{
Effects of practice on sex-related differences in performance on Embedded Figures
}

\author{
ALVIN G. GOLDSTEIN AND JUNE E. CHANCE \\ UNIVERSITY OF MISSOURI, COLUMBIA
}

\begin{abstract}
Men and women Ss were tested in an extended series of 68 Embedded Figures. The initial significant sex-related mean difference in discovery times was reduced to almost zero by the final trials. All Ss showed improvement with practice.
\end{abstract}

\section{Introduetion}

The extent to which an individual depends upon the visual field to orient himself in space is a problem which Witkin and his associates have been investigating for 20 years (Witkin, 1950; Witkin et al, 1954; Witkin et al, 1962). From this research has developed the polar concepts of field-dependence and field-independence, characterizing respectively, the behavior of individuals who are either more or less strongly affected by visual cues when making space oriented responses.

In its original conception, the field-dependence construct was defined by S's performance on several tasks involving visual-spatial orientation. The results of experiments employing these space orientation tasks were interpreted as evidence for important sex-related perceptual differences. For example, men, on the average, were considerably less field-dependent than were women. In a further development, Witkin demonstrated that performance in space orientation situations was consistent with performance on a non-space orientation task, the Embedded Figures Test (EFT; Witkin, 1950)。 In the EFT, which is a modification of Gottschaldt's test (in Ellis, 1950, p. 109-122), S is required to discover a simple figure which is perceptually embedded within a larger, complex figure. The test consisted of 24 items (see Witkin, 1950 for detailed description). The dependent variable was the time in seconds taken by $\mathrm{S}$ to discover the simple figure. The present experimental investigation was concerned with sex-related differences of field-dependency as measured by the EFT.

Witkin's data clearly showed within-session reduction of discovery time scores. Thus, for the men mean discovery time for the first 12 items was $54.5 \mathrm{sec}_{\text {. }}$, and for the last 12 items, $25.1 \mathrm{sec}$. Similarly, the women's means were 83.5 and 32.8 sec., respectively (Witkin, 1950, Table II, p. 10). These reductions, both significant at less than the .01 level, representing improvements of $53 \%$ for the men and $61 \%$ for the women, were not discussed by Witkin. Further evidence of the effect of practice upon EFT performance may be inferred from data obtained in a test-retest study spanning a three year period, where both mens' and womens' discovery time scores also decreased significantly (Witkin et al, 1954, p. 86). These data, which appear to be clear evidence of the long term effects of one practice session upon EFT performance were not discussed by Witkin in terms of their implication for the theory of field dependency.

There are at least two hypotheses to explain the sex related differences in discovery time. One, offered by Witkin, implies that the EFT is measuring the underlying personality trait of field-dependency, which is characterized as being a basic trait which is stable and strongly resistant to training (e. g., Witkin et al, 1954 , pp. $8-9,76,489)$. It is understandably difficult to reconcile this theoretical position with data suggesting within-session improvements in EFT discovery times. In contrast, these data are easily handled if it is assumed that the performance measured by the EFT is trainable. It follows, then, that if on the average men have had more relevant training than women, men's initial performance should be superior to women's initial performance. Furthermore, if it is not assumed that the asymptotic level of EFT skill differs between the sexes, then it also follows that women's total improvement should be greater than men's total improvement because women begin the task at a less advanced stage of training. It is also logically consistent to predict that with practice on EFT items the significant sex-related difference in performance will disappear. The purpose of the present experiment was to test that prediction.

\section{Method}

Twenty-four of the 68 EFT items used were from Witkin's original test; the remaining 44 items were taken from two different tests developed by Educational Testing Service.1 These tests have been shown to correlate between +.84 and +.56 with Witkin's EFT (Jackson et al, 1962). Although no two complex figures were identical, simple figures were repeated several times as only 14 simple figures were used. EFT items were administered exactly as reported by Witkin (1950). Elapsed time taken by $\mathrm{S}$ to make an incorrect tracing was not included in the discovery time measure. The maximum solution time permitted for each item was $2.5 \mathrm{~min}$. Presentation order of the $68 \mathrm{EFT}$ items was determined by random assignment prior to the beginning of the study. This order was reversed for half the Ss. Thirteen men and 13 women undergraduate college students were tested by a male $\mathrm{E}$ in two sessions separated by 48 hours. 


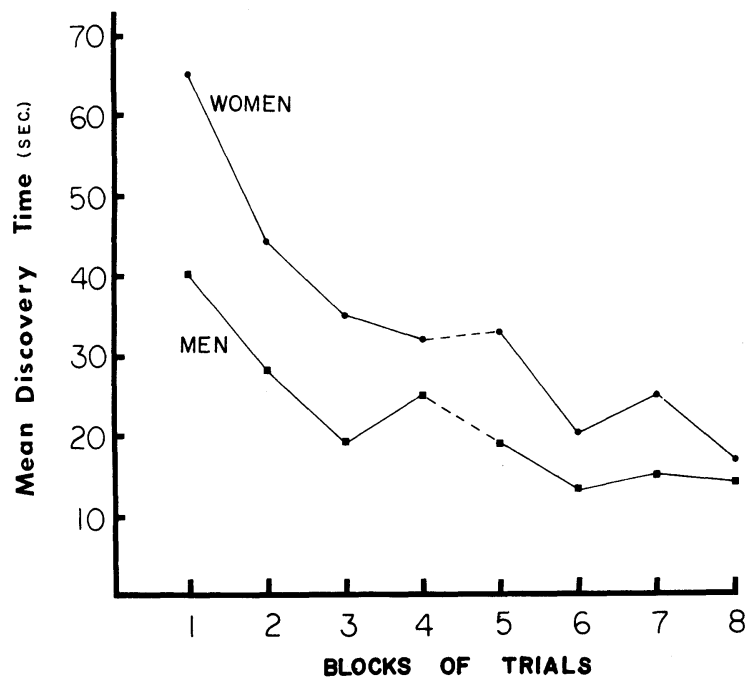

Fig. 1. Mean discovery time as a function of the number of Embedded Figure Items. Blocks 1 to 4 were completed in the first session: Blocks 5 to 8 in the second session.

\section{Results}

The data were grouped into eight blocks of mean discovery time scores as shown in Fig. 1. The first and eighth blocks are the means for all Ss on the first 10 and last $10 \mathrm{EFT}$ items. Each of the intervening blocks are means of 8 EFT items. Figure 1 graphically presents the discovery times for men and women as a function of the number of EFT items. The graphed data show that discovery times for men and women are inversely related to the number. of EFT items. From Blocks 1 to 8 the women decreased their discovery time scores by $74 \%$, and the men by $65 \%$. Furthermore, the sex-related difference in discovery time is 25 sec. in Block $1(t=2.4 ; p<.05)$ but is reduced to $3 \mathrm{sec}$. in Block $8(t<1.00)$. Finally, discovery time scores for all Ss decreased from Block 1 to Block 8 . In 21 of 26 cases, this decrease was greater than $50 \%$.

\section{Diseussion}

From the results of the present experiment, plus Witkin's original data (1950) and data of other reported studies (Jackson et al, 1962) it is quite clear that performance on the EFT rapidly improves, and learning is typical of all Ss. We have shown that although men and women may begin the EFT at significantly different levels of performance, after a series of practice trials, sex related differences are no longer reliable.

There are several implications of these data. If the EFT and body orientation measures are measuring the same thing, then there is a good possibility that field dependency is itself trainable and will respond to practice (see Witkin, 1948, for discussion of effects of practice). If, however, we find that body orientation is not trainable, then perhaps the EFT and orientation tasks are not measuring the same aspect of perceptual functioning. If we assume the EFT is measuring field dependency, then reduction in time scores must reflect a reduction in the influence of visual factors upon Ss. Unless another interpretation of the demonstrated improvement in EFT performance is offered, the concept of field dependency must be modified to include the effects of practice or training. Obviously, if a field dependent individual can become a field independent individual by simply being exposed to many EFT items, the power of the concept is indeed diminished.

Even if one treats the EFT merely as an empirical correlate of field dependency, the decrease in discovery times is not an attractive aspect of the test. Implicit in the use of a psychological test is the assumption that what is being measured is not continually and rapidly changing. Perhaps the test is most sensitive when $\mathrm{S}$ is first introduced to the task, or the test may measure different characteristics before and after practice (Jackson et al, 1962). Current research in the laboratory indicates that the EFT may be sensitive to interaction between sex of $E$ and sex of $S$. These are empirical questions, and until they are answered, the EFT should be used with caution. Speculating on this material, we would suggest that the reason for discovery time score differences between Ss is not primarily differential perceptual ability but is more likely differences in motivation to succeed in the task.

\section{References}

Ellis, W. D. A source book of Gestalt Psychology. New York: Humanities Press, 1950.

Jackson, D. N., Messick, S., \& Myers, C. T. The role of memory and color in group and individual embedded figures measures of field independence. Res. Bull., 1962, 62-34, Princeton, N. J.: Educational Testing Service.

Witkin, H. A. The effect of training and of structural aids on performance in three tests of space orientation. C. A. A. Report No. 80, Washington, D. C., 1948.

Witkin, H. A. Individual differences in ease of perception of embedded figures. J. Pers., 1950, 19, 1-15.

Witkin, H. A., Lewis, H. B., Hertzman, M., Machover, K., Meissner, P. B., \& Wapner, S. Personality through perception. New York: nHarpers, 1954.

Witkin, H. A., Dyk, R. B., Faterson, H. F., Goodenough, D. R., \& Karp, S. A. Psychological differentiation. New York: Wiley, 1962.

Note

1. Education Testing Service Form K-JETQ3, 1961; and Form JETQ4, 1962. 